\title{
BMJ Open Role of institutional entrepreneurship in building adaptive capacity in community-based healthcare organisations: realist review protocol
}

Sweatha lyengar, ${ }^{1}$ Aaron Katz, ${ }^{2}$ Jo Durham ${ }^{1}$

To cite: lyengar S, Katz A, Durham J. Role of institutional entrepreneurship in building adaptive capacity in community-based healthcare organisations: realist review protocol. $B M J$ Open 2016;6:e010915. doi:10.1136/bmjopen-2015010915

- Prepublication history and additional material is available. To view please visit the journal (http://dx.doi.org/ 10.1136/bmjopen-2015010915).

Received 18 December 2015 Revised 25 February 2016 Accepted 29 February 2016

\section{CrossMark}

${ }^{1}$ Faculty of Medicine and Biomedical Sciences, School of Public Health, The University of Queensland, Herston, Queensland, Australia

${ }^{2}$ Department of Health Services, School of Public Health, University of Washington, Seattle, Washington, USA

Correspondence to Dr Jo Durham; m.durham@uq.edu.au

\section{ABSTRACT}

Introduction: Over the past 3 decades, there has been a substantial shift to the marketisation of governmentfunded health services. For organisations traditionally buffered from the competitive pressures of for-profit enterprises, such as community-based organisations, this means developing the capacity to adapt to competitive tendering processes, shifting client expectations, and increasing demands for greater accountability. Drawing on ideas of institutional entrepreneurship, we believe that attempts to build adaptive capacity require the transformation of existing institutional arrangements. Key in this may be identifying and fostering institutional entrepreneurs-actors who take the lead in being the impetus for, and giving direction to, structural change. This study focuses on the strategies used by institutional entrepreneurs to build adaptive capacity in the community-based healthcare sector.

Methods and analysis: The research will use an adapted rapid realist review. The review will find underlying theories that explain the circumstances surrounding the implementation of capacity-building strategies that shape organisational response and generate outcomes by activating causal mechanisms. An early scoping of the literature, and consultations with key stakeholders, will be undertaken to identify an initial programme theory. We will search for relevant journal articles and grey literature. Data will be extracted based on contextual factors, mechanisms and outcomes, and their configurations. The analysis will seek patterns and regularities in these configurations and will focus on confirming, refuting or refining our programme theory.

Ethics and dissemination: The study does not involve primary research and, therefore, does not require formal ethical approval. However, ethical standards of utility, usefulness, feasibility, propriety, accuracy and accountability will be followed. The results will be written up according to the Realist and Meta-Review Evidence Synthesis: Evolving Standards guidelines. Once completed, findings will be published in a peer-reviewed journal.

Trial registration number: CRD42015026487.
Strengths and limitations of this study

- A realist review is rich in explanatory power and builds an understanding of how and why interventions work in complex systems such as health markets.

- The engagement of a reference group, consisting of industry experts and researchers, will ensure complete coverage of the literature, resulting in a powerful programme theory.

- Realist reviews are harder to reproduce, as relationships are theorised, often based on judgement, intuition and experience.

\section{BACKGROUND}

In most developed nations, there has been a substantial shift to the marketisation of government-funded health services. ${ }^{1}{ }^{2}$ In every part of the health system, whether nominally public or nominally private, healthcare - professional services, social and welfare services, education, training, drugs, case management, and decent, humane treatmentcan be bought and sold. ${ }^{3}$ Patients are customers, health is a commodity, and the consumption of goods and services takes place through voluntary exchanges in the market. ${ }^{1}$ While the broader political agenda oriented towards improving service delivery, maximising consumer choice, and ensuring effective, equitable, responsive and efficient services, is well understood, the commodification of health is, fundamentally, an issue concerning organisational, rather than simply political, sustainability. ${ }^{2} 4$ The shift to a market approach represents a significant reform process that, ultimately, aims to transform the way in which health services are delivered and consumed. ${ }^{2}$ For organisations traditionally buffered from the competitive pressures of for-profit enterprises, such as not-for-profit and community-based organisations (CBOs), the journey into a competitive market is 
arduous and uncertain. Providers are having to develop the capacity to adapt to competitive tendering processes, shifting client expectations, and increasing demands for greater accountability. The ability of CBOs to adapt and respond in this changing political environment is crucial not only for business continuity, but also for the growth of these organisations. Despite the crucial role that CBOs play in the communities they serve, there is very limited understanding about how and in what circumstances these organisations adapt in anticipation of, and in response to, exogenous shocks that challenge existing institutional arrangements. Drawing on ideas of institutional entrepreneurship, we believe that attempts to build adaptive capacity require the transformation of existing institutional practices. Key in this may be identifying and fostering institutional entrepreneurs, actors who take the lead in being the impetus for, and giving direction to, structural change. In this research, we draw on ideas from the theory of institutional entrepreneurship to examine how change agents may engage in reshaping existing institutional practices in order to build an organisation's adaptive capacity. ${ }^{5-10}$ A full glossary of terms is provided in online supplementary file 1 .

\section{What is adaptive capacity, and why is it important?}

Researchers have begun to recognise that the ability to challenge the status quo, that is, established ways of thinking and doing things, and to successfully adopt more effective processes, is a distinct form of organisational capacity: adaptive capacity. ${ }^{11}{ }^{12}$ In this paper, we define adaptive capacity as the quest for change in pursuit of not only a continuity of core functions, but also a transformation of these functions in order to capitalise on opportunities for change. ${ }^{11} 13{ }^{14}$ It is this notion of adaptive capacity which resonates with Bains and Durham, who suggest that this transformational view of adaptive capacity necessarily includes 'concepts of renewal, regeneration and re-organisation', requiring CBOs to act as learning organisations. ${ }^{13}$ Adaptive capacity is, therefore, a dynamic process, embedded in all aspects of day-to-day activities. An organisation with adaptive capacity will critically reflect on new opportunities and knowledge, and harness this new knowledge to accommodate for growth. For this to happen, however, it requires a mix of key attributes: innovation, flexibility, awareness, change readiness, systems thinking, social capital, strong networks and leadership. ${ }^{11-13}$

Available data suggests that CBOs are finding it increasingly difficult to face changes in the political environment in which they operate. ${ }^{11}{ }^{15}$ In a sector traditionally buffered from the competitive pressures of for-profit enterprises, CBOs are having to become more adaptive to changes in their operating environments in the form of changing circumstances, service demands and client expectations. These organisations increasingly face adaptive challenges requiring them to reject the familiar and, instead, develop the capacity to harness creativity and knowledge to fashion innovative responses, integrate lessons learned, and embrace transformational change. ${ }^{11} 14$ The increasing importance of understanding adaptive capacity in CBOs has led to the identification of a key gap in the literature: how and in what circumstances can adaptive capacity be built in CBOs? Key in this may be identifying and fostering institutional entrepreneurs.

\section{Institutional entrepreneurs in building adaptive capacity}

Building adaptive capacity in CBOs can be difficult. Institutional theory suggests that institutions are resilient social structures, in which deeply embedded beliefs and patterns of behaviour govern the appropriate conventions of its constituents. ${ }^{5}{ }^{6}$ Deeply rooted sources of resistance to change in healthcare, for example, are well documented, in that practitioners and managers who are directly affected will resist change. ${ }^{6}{ }^{14}$ Similarly, nonprofits and $\mathrm{CBOs}$ are often resistant to dominant market-based approaches that directly challenge their not-for-profit mission. Institutional entrepreneurship focuses on the nature of these exogenous shocks that challenge existing institutional arrangements. It suggests that some actors may be better placed than others to shape institutions despite pressures towards stasis. ${ }^{10}$ Institutional entrepreneurs are defined as 'actors who serve as catalysts for structural change and take the lead in being the impetus for, and giving direction to, change'. ${ }^{9}$ They are actors with sufficient resources to create new institutions that promote their particular interests. Studies suggest that key to the institutional entrepreneur's scope to enact institutional change is his or her legitimacy in the organisation, social position in the field, social capital and formal authority. ${ }^{7-9} 16 \quad 17$ According to Bains and Durham, 'institutional entrepreneurs have strong leadership skills although they may work invisibly: connecting; spanning boundaries; mobilizing resources and keeping alive a strategic focus'. ${ }^{13}$

We propose that the theory of institutional entrepreneurship provides a powerful theoretical lens for understanding the processes of building adaptive capacity. However, there is limited research that explains how and in what circumstances institutional entrepreneurs could enact institutional change in order to build adaptive capacity in CBOs. Furthermore, while there is some research available on the process of institutional entrepreneurship in the health arena, the research that is available provides limited practical guidance for $\mathrm{CBO}$ and institutional entrepreneurs on how to build adaptive capacity. In this review, our objective is to understand the circumstances surrounding the implementation of capacity-building strategies that shape organisational response and generate outcomes by activating causal mechanisms. The information gathered can bolster successful CBO adaptation to better serve the communities they support.

\section{METHODS}

\section{Realist review}

The research questions will be addressed using a realist review. ${ }^{18-23}$ While positivist approaches to understanding 
interventions, such as Cochrane-style systematic reviews and meta-analyses, can provide causal models to inform 'what works' by focusing on differences in outcomes between groups, they fail to recognise the complexities of social context and cannot elucidate the underlying processes that explain 'how it works', 'why it works' and 'in what context it works'. ${ }^{24-26}$ Against this logic, realism is typically used to understand complex interventions, such as capacity building, in complex, interactive health systems with substantial heterogeneity. Capacity-building strategies are highly contextual; the outcome of the 'same' strategy will vary 'depending on who delivers it, to which learners, in which circumstances and with which tools and techniques'. ${ }^{27}$ Hence, a strategy that is successful in one setting may be unsuccessful in another setting. In such complex systems, the outcomes depend on individuals making decisions in a semipredictable (demiregular) manner about how to use the resources available to them in particular contexts. Thus, the act of restricting the scope of the review to 'what works' by only focusing on the outcomes, destroys the intricate relationships and underlying processes which we are seeking to understand. ${ }^{20}$

Realist review is an interpretative, theory-driven approach that uses qualitative, quantitative and mixedmethods research evidence to understand and explain how mechanisms $(\mathrm{M})$ produce different outcomes $(\mathrm{O})$ in different contexts $(\mathrm{C}) .^{2025} 28$ At the heart of realist review lies the concept of generative causation, represented below in the basic realist formula:

$$
\text { Context }(\mathrm{C})+\text { mechanism }(\mathrm{M})=\operatorname{outcome}(\mathrm{O})
$$

Realist review is based on the principle that a capacitybuilding strategy does not trigger change; it is the mechanisms underlying the strategy that generate outcomes. ${ }^{22}{ }^{28-30}$ It is this notion of programme "mechanisms' that takes a step away from asking whether a strategy 'works' to understanding what it is about a strategy which makes it 'work'. Mechanisms refer to the elements in the cognitive decision-making process, such as collective beliefs, norms, preferences, which interact with resources, opportunities and constraints to influence changes in behaviour. ${ }^{19} 20232428$ Taking capacitybuilding strategies used by institutional entrepreneurs as an example, such strategies, such as providing information to senior management about imminent policy threats may motivate senior management staff to make an internal change in service provision, whereby the mechanism is the decision or reasoning to make an internal change in service provision (forward planning), rather than the strategy itself (providing information to senior management).

These mechanisms, such as forward planning, are enabled or disabled by the context in which the intervention is implemented. This is because similar mechanisms may be triggered in some contexts, producing similar patterns of behaviour, or triggered in other contexts, producing different patterns of behaviour. Context may refer to broad cultural, social, historical or institutional features (eg, the degree of heterogeneity and institutionalisation within the not-for-profit organisation) to features affecting the implementation of the strategy (eg, whether there is adequate funding within the organisation). It could also relate to the conditions in which individuals seek to enact their choices (eg, senior management will be more likely to enact change in a context in which institutional entrepreneurs are perceived as legitimate). ${ }^{2} 2324$ Drawing once again on examples from the current research, a resilient culture within the organisation is a contextual condition which may disable the activation of mechanisms that lead to institutional change.

Outcomes can be intended or unintended, intermediate or final. ${ }^{223}$ In the current research, the intended outcome of interest is adaptive capacity (the ability to monitor, assess, respond to and stimulate change), which necessarily includes concepts of shared understanding and purpose, strategic alliances with other organisations, willingness to question accepted ways of working, and innovative approaches to service provision. While adaptive capacity-building strategies are implemented with these desired outcomes in mind, the variations in contexts and mechanisms means that strategies are liable to have mixed outcome patterns. ${ }^{27} 30$ For example, the way in which senior management react to a particular message may depend on the organisational culture. For example, the institutional entrepreneur may propose a change in service provision. Senior management from a CBO less institutionalised (established norms and patterns of behaviour) may react to this message exactly as institutional entrepreneurs expect and take strategic action. Some senior management, from a more institutionalised organisation, may find the same message threatening to existing institutional arrangements and may dismiss the message altogether. It is also worth noting that an outcome of one strategy can also become a context or mechanism that provides another outcome, creating a 'ripple effect'. ${ }^{31}$ For instance, using the example provided previously, the capacity to forward plan may become a contextual factor in the next context-mechanism-outcome $(\mathrm{CMO})$ configuration, that is, senior management will be more likely to enact change in a context in which the organisation engages in forward planning.

\section{Research aim and objectives}

The primary aims of this realist review are (1) to understand how institutional entrepreneurs build adaptive capacity in CBOs and (2) to test and refine underlying theories that explain 'what works, for whom, and in what circumstances'. Drawing on previous work in institutional entrepreneurship and organisational adaptive capacity, the reviewers will focus on synthesising the evidence to identify CMO configurations that explain the contexts in which particular mechanisms generate particular outcomes. 
An additional, secondary aim concerns the interface between research, policy and practice. The results will be useful to policymakers, CBO institutional entrepreneurs, and academics in understanding how to effectively build a CBO's adaptive capacity. The findings will provide policymakers with explanations that are sensitive to the context (ie, in situations like $\mathrm{X}$, use strategy $\mathrm{Y}$ and keep an eye out for Z). ${ }^{25}$ Therefore, policymakers are more likely to be able to interpret an explanation of why a strategy works better in one organisation than in another.

Our overarching research question is:

1. What strategies, contextual factors and mechanisms are necessary for institutional entrepreneurs in CBOs to build adaptive capacity?

a. In what ways do institutional entrepreneurs in CBOs create a vision and mobilise resources to enact change and build adaptive capacity?

b. What is the role of enabling conditions, including field-level determinants and individual-level determinants, in adaptive capacity-building efforts?

c. What are the key mechanisms or social processes that influence or drive the successful building of adaptive capacity?

d. How do key mechanisms and enabling conditions interact to produce change?

The review will follow the steps of a rapid realist review (RRR), as proposed by Saul et al. ${ }^{21}$ The RRR process streamlines the review process by engaging knowledge users and review stakeholders to rapidly identify relevant documents for review, resulting in a review within 3-6 months. However, stakeholder involvement does not replace a literature search; rather, it provides a method to quickly identify relevant material for tailoring the search strategy and recommending CMO configurations. The RRR process is particularly useful if there is a small evidence base. A limitation of the RRR process is that it bypasses the initial identification of CMO configurations, which may limit the generalisability and potency of findings. Therefore, the study will use an adapted RRR process, moving between the following steps: drawing on external stakeholder expertise, describing the initial hypotheses or relevant programme theories, undertaking a thorough search of the literature for relevant papers, appraising the evidence, synthesising the data, and interpreting the evidence to test and refine our theoretical framework.

\section{Study design}

\section{Step 1: identify potential theories}

The initial identification of a rough programme theory is the first step to developing an overall, more powerful programme theory of how institutional entrepreneurs build adaptive capacity. ${ }^{20}$ Central to the realist review is developing and refining this candidate theory. The initial theory is a preliminary sketch of what it is the research question is investigating, whereas, the revised programme theory describes what a programme, or in this case, a strategy, is expected to do and how it is expected to work. We are looking for substantiative theories, existing theories within particular disciplines that explain the process of institutional entrepreneurship, but also how institutional entrepreneurs might work to build adaptive capacity in CBOs. We will use a variety of methods to derive our list of theories, including brainstorming within the review team, scoping the literature, and consulting individual experts in the field.

To date, the concept of institutional entrepreneurship has been commonly applied to institutional change at the organisational level. An initial scoping review of the literature uncovered a range of issues and areas concerning the enabling conditions for, and the process of, institutional entrepreneurship. Research suggests that the work undertaken by institutional entrepreneurs is inevitably contingent on prevailing forms of field-level and individual-level determinants. ${ }^{5}$ External pressures and crises, in the form of social upheaval, political pressures, technological disruptions, regulatory changes and competitive discontinuities, are identified by Child et $a l^{33}$ and Greenwood et $a l^{34}$ as field-level enabling conditions for institutional entrepreneurship, as they disrupt existing institutional arrangements, motivating individuals to reconsider the status quo. Another important field-level determinant is the structure of the organisation, that is, the degree of heterogeneity and institutionalisation. Heterogeneous institutional arrangements (variance in the characteristics of institutional processes) and lower degrees of institutionalisation (established norms and patterns of behaviour) are likely to give rise to institutional incompatibilities, driving individuals to question existing arrangements and take strategic action as institutional entrepreneurs. ${ }^{535}$

Although field-level determinants play an important role in enabling institutional entrepreneurship, 'only some actors will exploit the opportunity to become institutional entrepreneurs. ${ }^{5}$ Therefore, individual-level determinants, including the social position of an actor, also play an enabling role in institutional entrepreneurship. Social position-the position of an individual in the structure of social networks-might affect both actors' perception of a field and their access to resources needed to engage in institutional change. ${ }^{36}$ Research suggests that actors at the centre of organisations are confined by the institution which prevents them from recognising alternative processes, whereas actors at the margins of organisations are less embedded in organisational practises, thus prompting them to make change. ${ }^{6} 10$

In the literature, two main processes of institutional entrepreneurship that have received considerable attention are (1) creating vision and (2) mobilising resources. Institutional entrepreneurs must craft a vision for change in such a way that it appeals to the widest possible audience of potential allies. According to Rao et $a l^{37}$ 'institutional entrepreneurs can mobilize legitimacy, finances, and personnel only when they are able to 
frame the grievances and interests of aggrieved constituencies, diagnose causes, assign blame, provide solutions, and enable collective attribution processes to operate'. This necessarily includes specification, exposing organisational failings, and justification, proposing solutions as superior to previous arrangements. ${ }^{9}$ Because they can seldom transform institutions alone, institutional entrepreneurs must mobilise key constituents with a diverse range of social skills depending on the kind of change they intend to enact. ${ }^{38}$ According to Leca et al, ${ }^{9}$ 'tangible resources such as financial assets can be used during early stages of the process to bypass the sanctions likely to be imposed on the institutional entrepreneur who questions the existing institution by opponents of the proposed change'. Furthermore, intangible resources, such as social capital, legitimacy and formal authority, can enable institutional entrepreneurs to be taken seriously by allies. These findings from the initial scoping review will contribute to the formation of our initial programme theory.

Using the online Delphi technique, we will consult with key stakeholders in an expert reference group, including academics, CBO member representatives, managers, staff and public servants, to assist us in the identification of relevant articles and documents for inclusion in the review. The Delphi technique is used to prompt reflection and discussion among a group of experts with the aim of reaching consensus about the programme theory. ${ }^{39}$ The reference group will act as a 'reality check' to test our emerging understandings of the programme theory. ${ }^{40}$ The authors will meet regularly with the stakeholder group and will also communicate via email.

\section{Step 2: search strategy}

Following the Realist and Meta-Review Evidence Synthesis: Evolving Standards (RAMESES) guidelines for a realist review, which recognises the limitations of fixed search protocols, we will undertake an iterative search of the literature with a broad focus that responds flexibly to emerging findings. ${ }^{19} 25$

The initial search strategy will involve two phases: (1) we will search for research evidence that explains how institutional entrepreneurs facilitate institutional change and build adaptive capacity in community-based, nonprofit organisations and (2) we will seek additional data to test and refine our programme theory. Table 1 provides preliminary search terms based on key concepts provided by the research team; the search terms will be iteratively narrowed based on the relevance of the retrieved documents, inclusion and exclusion criteria, and the extent to which each study clarifies the CMO configurations. The literature will be searched using combinations of these key terms in English and their truncations from 1988, the year institutional entrepreneurship was introduced by DiMaggio, to the present. We anticipate our search strategy to include databases such as PubMed, Web of Science, PsycINFO, Scopus, EMBASE, EconLit, the Cochrane Library, CINAHL, JSTOR, Emerald, Google, and any other relevant databases identified by the team (these databases were selected as they offer extensive indexing of the literature). This search will be supplemented with bibliographic searches of reference lists in identified documents using the snowballing technique to identify additional documents. Grey literature, including evaluation reports and policy documents by governments, organisations and consultancy firms, as well as dissertations and theses, will also be included in the search. Searching for new documents will end at the point of theoretical saturation; that is, when there is sufficient evidence to claim that the revised programme theory is plausible. Documents meeting inclusion criteria will be compiled in endnote.

\section{Step 3: study selection criteria and procedures}

Documents will be selected for the review based on what new knowledge they bring to our thinking about the

Table 1 Search strategy

\begin{tabular}{|c|c|}
\hline Search number $\mathrm{Se}$ & arch terms \\
\hline EMBASE platform & \\
\hline 1 & Institutional NEXT/1 entrepreneur* \\
\hline Scopus platform & \\
\hline 1 & TITLE-ABS-KEY (institutional PRE/1 entrepreneur ${ }^{\star}$ ) \\
\hline 2 & $\begin{array}{l}\text { (TITLE-ABS-KEY (ngos) OR TITLE-ABS-KEY (ngo) OR TITLE-ABS-KEY (engo) OR TITLE-ABS-KEY } \\
\left.\text { (engos) OR TITLE-ABS-KEY ('Community Base }{ }^{\star \prime}\right) \text { OR TITLE-ABS-KEY ('Not for profit') OR } \\
\left.\text { TITLE-ABS-KEY ('Non profit') OR TITLE-ABS-KEY ('non profit }{ }^{\star \prime}\right) \text { ) }\end{array}$ \\
\hline 3 & $\begin{array}{l}\text { (TITLE-ABS-KEY (institutional PRE/1 entrepreneur*)) AND ((TITLE-ABS-KEY (ngos) OR } \\
\text { TITLE-ABS-KEY (ngo) OR TITLE-ABS-KEY (engo) OR TITLE-ABS-KEY (engos) OR TITLE-ABS-KEY } \\
\text { ('Community Base*') OR TITLE-ABS-KEY ('Not for profit') OR TITLE-ABS-KEY ('Non profit') OR } \\
\text { TITLE-ABS-KEY ('non profit'))) }\end{array}$ \\
\hline Web of Science pla & tform \\
\hline 1 & $\begin{array}{l}\text { (('institutional entrepreneur") AND (NGO OR NGOs OR ENGOs OR ENGO OR 'Community base*' } \\
\text { OR 'Not for profit'” OR nonprofit')) }\end{array}$ \\
\hline
\end{tabular}

${ }^{*}$ The above searches will be modified for other databases. Search terms are highlighted in bold text in the search result, as well as in subsequent articles viewed from the search. 
programme theory and the extent to which they can refine CMO configurations. This is likely to include editorials, opinion pieces, evaluations, programme manuals, reviews and commentaries. Focus will be placed on aspects of the document that relate to contexts, mechanisms and outcomes which contribute to our understanding of how institutional entrepreneurs transform CBOs to build adaptive capacity. Based on discussions between the reviewers (SI, JD and AK), the inclusion criteria will include papers related to the process of institutional entrepreneurship in communitybased, non-profit settings, in any country. The inclusion criteria may extend to papers in CBOs outside of healthcare, if insufficient papers are found that relate specifically to community-based health services.

In the first stage of searching, SI will screen the title, abstract and keywords against the broad inclusion criteria outlined in table 2. Articles meeting the inclusion criteria will be obtained for full-text screening. A random sample of $10 \%$ of documents will be selected, assessed and discussed by all review authors. It is expected that a number of documents will require discussion between the reviewers to decide whether to integrate the paper into the review. Documents meeting inclusion criteria will proceed to data extraction.

\section{Step 4: data extraction}

Realist reviews typically synthesise information by notetaking and annotation rather than using a standardised list of questions as used in a traditional systematic review. The explanatory accounts will initially be tabulated in an Excel spreadsheet, and will be examined for study characteristics (eg, sample size, setting, study objectives), as well as information on how, why and in what contexts institutional entrepreneurs impose institutional change in order to build capacity in CBOs. During extraction, aspects of each paper will be assessed for relevance based on various factors, including definitions, theoretical frameworks employed, strategies and processes, mechanisms, contexts and outcomes. Using a similar approach to Pearson et al, ${ }^{41}$ we will seek explanatory accounts in the form of 'If...then' statements to identify potential contexts and mechanisms. For example, if institutional entrepreneurs are perceived as legitimate entities within the institution, then intended targets are more likely to believe that the organisation is at risk. However, as explanatory accounts may not always report contexts and mechanisms in a consistent format, we will also seek standalone accounts of each of these elements. In a second phase, the NVivo qualitative software will be used for coding; that is, to index and link relevant explanatory accounts in an iterative manner allowing authors to identify inter-relationships and overlaps before further development. ${ }^{42}$

Relevance and rigour of papers will be assessed using the Mixed Methods Appraisal Tool which has theoretical and content validity, and has been tested for efficiency and reliability. ${ }^{43}$ The tool will only be applied to pertinent aspects of the studies that relate to our programme theory, rather than the studies as a whole. To ensure transparency in the data extraction process, we will develop a summary table specifying the publication title, authors, year of publication, objectives, type of study, setting and different methodological aspects.

\section{Step 5: data synthesis}

The initial candidate theory identified in step 1 will be used as a basis to analyse the data. Using a mix of inductive and deductive analytical processes, explanatory accounts, which will be coded within NVivo during the extraction phase, will be examined to see if they confirm, refute, or refine our candidate theory, that is; is the account novel, and does it add anything to our understanding of the programme theory? We will consolidate the explanatory accounts to develop the final programme theory which will be to determine what it is about institutional entrepreneurship that works to build adaptive capacity, and for whom, in what circumstances, in what respects, and why. We will seek to determine if the extracts infer novel accounts of what the causal mechanisms might be, the contexts in which the mechanisms might be triggered, or the outcomes of

Table 2 Inclusion criteria

\section{Description}

1 Document discusses the process of institutional entrepreneurship as it relates to transformation and change and is implicitly or explicitly underpinned by institutional theory

2 Document is about the capacity of an organisation/institution to transform, including the ability to adapt and capitalise on opportunities for change

3 Document describes a community-based, non-profit organization(s) that provides healthcare services or public services to individuals

$4 \quad$ Document discusses at least one of the following factors that will contribute to the synthesis of our emerging programme theory:

The strategies used by institutional entrepreneurs in transformative efforts to build adaptive capacity

The outcomes of the strategies they employ

The beliefs, norms, values, preferences, and cognitive processes that influence behaviour

The circumstances/conditions in which these different outcomes are generated 
these mechanisms. While reading the explanatory accounts and in our attempt to synthesise the information alongside existing theory into a consolidated explanatory account (programme theory), we will consider: Is this account novel? Does this account add to our understanding of contexts, mechanisms, and outcomes mentioned in related accounts? In terms of expressing CMO configurations, we will ask: Does the consolidated explanatory account adequately reflect the contexts, mechanisms, outcomes and their configurations, proposed in the underlying explanatory accounts? Following the RAMESES guidelines, the final programme theory will be summarised through a narrative synthesis of the interaction between context, mechanism and outcome, using a logical model where appropriate.

\section{Validity}

A number of criteria will be met in establishing the validity of the review. First, the iterative process of understanding how institutional entrepreneurs build adaptive capacity in CBOs, will require the reviewers to move between empirical data and formulating CMO configurations, which will enhance internal validity. The deliberate inclusion of context in the analysis will increase the generalisation of our programme theory. Further, the use of an expert stakeholder group to provide insight, feedback and to review the findings will also contribute to validity.

\section{Ethics}

The study does not involve primary research and, therefore, does not require formal ethical approval. However, ethical standards of utility, usefulness, feasibility, propriety, accuracy and accountability will be followed. ${ }^{44}$

\section{Dissemination}

The results of the review will be written up according to the RAMESES guidelines. An international collaborative study providing methodological guidance and reporting standards for a realist review is available online. ${ }^{22} 23$ Findings will be published in a peer-reviewed journal.

\section{DISCUSSION}

Increasingly, government-funded health services are shifting to market-driven approaches. The need to examine the influence of policy forces that are changing existing institutional arrangements is becoming even more urgent. Rigorous research is needed to identify how and in what circumstances adaptive capacity can be built in CBOs so that they have a better chance of surviving and thriving in changing environments. Key to this, is identifying the role of institutional entrepreneurs in helping CBOs to reshape existing institutional practices in order to capitalise on opportunities for change. While there is some research on the process of institutional entrepreneurship, there is limited understanding of how institutional entrepreneurs enact institutional change in order to build adaptive capacity. By capturing the relationship between context, mechanism and outcome, the findings of this review will provide valuable transferrable lessons in 'what strategies work, for whom, in what circumstances, to what extent', and most importantly 'how and why?'. For CBOs wishing to position themselves for adaptability in light of recent policy changes, the findings of this research may help in identifying capacity-building strategies and possible focal points for both decision-makers and institutional entrepreneurs. A better understanding of how CBOs adapt and respond to market-driven policy forces is critical to meeting Government's objectives of using CBOs in the health sector to deliver public, demand-driven services to their communities.

There are strengths and limitations to using a realist review. Unlike a systematic review that explicitly attempts to control context, a realist review is rich in explanatory power and builds an understanding of how and why interventions work. Instead of providing a judgement on whether certain capacity-building strategies used by institutional entrepreneurs are 'good' or 'bad', the realist review will explain 'what strategies work, for whom, in what circumstances, to what extent', and 'how and why?'. However, compared to systematic reviews, realist reviews are harder to reproduce as relationships are theorised, often based on judgement, intuition and experience. To minimise this limitation, we will include a summary table and methodological details of papers included in this review. Furthermore, often context and mechanism details are lacking, limiting the information that can be extracted from documents. In these instances, the engagement of the expert reference group serves to explore possible CMO configurations in relation to current experiences and other relevant literature.

Acknowledgements The authors would like to thank the reviewers for their invaluable comments.

Contributors SI and JD conceived and designed the study. SI drafted the first manuscript with AK and JD revising for important intellectual content. All authors have given final approval of the version to be published.

Funding This research received no specific grant from any funding agency in the public, commercial or not-for-profit sectors.

Competing interests None declared.

Provenance and peer review Not commissioned; externally peer reviewed.

Open Access This is an Open Access article distributed in accordance with the Creative Commons Attribution Non Commercial (CC BY-NC 4.0) license, which permits others to distribute, remix, adapt, build upon this work noncommercially, and license their derivative works on different terms, provided the original work is properly cited and the use is non-commercial. See: http:// creativecommons.org/licenses/by-nc/4.0/

\section{REFERENCES}

1. Davidson B. Contestability in human services markets. J Austr Polit Econ 2011;68:213-39.

2. Durham J, Bains A. Research protocol: a realist synthesis of contestability in community-based mental health markets. Syst Rev 2015;4:32. 
3. Health, U.N.M.P.T.F.o.C., H. Maternal, and U.N.M. Project. Who's got the power? Transforming health systems for women and children. London: Earthscan, 2005.

4. Boychuck GW. The changing political and economic environment of health care in Canada. Discussion Paper no. 1. Ottawa: Commission on the Future of Health Care in Canada, 2002.

5. Battilana J, Leca B, Boxenbaum E. How actors change institutions: towards a theory of institutional entrepreneurship. Annals 2009;3:65-107.

6. Lockett A, Currie G, Waring J, et al. The role of institutional entrepreneurs in reforming healthcare. Soc Sci Med 2012;74:356-63.

7. Bruton GD, Ahlstrom D, Li H-L. Institutional theory and entrepreneurship: Where are we now and where do we need to move in the future? Entrep Theory Pract 2010;34:421-40.

8. Williams CC, Shahid MS. Informal entrepreneurship and institutional theory: explaining the varying degrees of (in)formalization of entrepreneurs in Pakistan. Entrepreneurship and Regional Development, 2014:1-25.

9. Leca B, Battilana J, Boxenbaum E. Agency and institutions: a review of institutional entrepreneurship. Working Paper 08-096. Harvard Business School. [Working Paper 08-096] 2008. http://citeseerx.ist. psu.edu/viewdoc/download?doi=10.1.1.461.6523\&rep=rep1\& type $=$ pdf

10. Lockett A, El Enany N, Currie G, et al. Health services and delivery research, in a formative evaluation of Collaboration for Leadership in Applied Health Research and Care (CLAHRC): institutional entrepreneurship for service innovation. Southampton, UK: NIHR Journals Library, 2014.

11. Sussman C. Building adaptive capacity: the quest for improved organizational performance. Boston, MA: Management Consulting Services, 2004. http://wikiciv.org.rs/images/5/5d/Sussman_(2004) Building_Adaptive_Capacity.pdf

12. Mcmanus S, Seville $\mathrm{E}$, Brunsdon D, et al. Resilience management: a framework for assessing and improving the resilience of organisations. New Zealand: Resilient Organisations Programme, 2007. http://cpor.org/ro/ResilienceMgmtResearch(2007).pdf

13. Bains A, Durham J. Community-based organisations: developing organisational resilience to build back better. 3rd International Conference on Building Resilience 2013. 17-19 September, 2013, Ahungalla, Sri Lanka, (1-12). Hill and Associates.

14. Schein EH. Organizational psychology. Englewood Cliffs, NJ: Prentice-Hall, 1980.

15. W.P. The new landscape for nonprofits. Boston: Harvard Business School Publishing Corporation, 1999:127-36.

16. Breton M, Lamothe L, Denis JL. How healthcare organisations can act as institutional entrepreneurs in a context of change. $J$ Health Organ Manag 2014;28:77-95.

17. Eriksson N. Ujvari S. Fiery spirits in the context of institutional entrepreneurship in Swedish healthcare. J Health Organ Manag 2015;29:515-31.

18. Greenhalgh T, Wong G, Westhorp G, et al. Protocol-Realist and meta-narrative evidence synthesis: evolving standards (RAMESES). BMC Med Res Methodol 2011;11:115.

19. Pawson R. Evidence-based policy: a realist perspective. London: SAGE, 2005.

20. Pawson R, Greenhalgh T, Harvey G, et al. Realist review-a new method of systematic review designed for complex policy interventions. J Health Serv Res Policy 2005;10(Suppl 1):21-34.
21. Saul JE, Willis CD, Bitz J, et al. A time-responsive tool for informing policy making: rapid realist review. Implement $\mathrm{SCi}$ 2013;8:103.

22. Wong G, Greenhalgh $\mathrm{T}$, Westhorp $\mathrm{G}$, et al. RAMESES publication standards: realist syntheses. BMC Med 2013;11:21.

23. Wong G, et al. Realist Synthesis: Rameses training materials 2013.

24. Durham J, Blondell SJ. Research protocol: a realist synthesis of cross-border patient mobility from low-income and middle-income countries. BMJ Open 2014;4:e006514.

25. Best A, Greenhalgh T, Lewis S, et al. Large-system transformation in health care: a realist review. Milbank $Q$ 2012;90:421-56.

26. Berwick DM. The science of improvement. JAMA 2008;299:1182-4.

27. Wong G, Greenhalgh T, Westhorp G, et al. Realist methods in medical education research: what are they and what can they contribute? Med Educ 2012;46:89-96.

28. Pawson R. The science of evaluation: a realist manifesto. London: SAGE, 2013.

29. Astbury B, Leeuw FL. Unpacking black boxes: mechanisms and theory building in evaluation. Am J Eval 2010;31:363-81.

30. Pawson R, Tilley N. Realistic evaluation. Thousand Oaks, CA, London: Sage, 1997.

31. Jagosh J, Bush PL, Salsberg J, et al. A realist evaluation of community-based participatory research: partnership synergy, trust building and related ripple effects. BMC Public Health 2015;15:725

32. Wong G, Greenhalgh T, Pawson R. Internet-based medical education: a realist review of what works, for whom and in what circumstances. BMC Med Educ 2010;10:12.

33. Child J, Lu Y, Tsai T. Institutional entrepreneurship in building an environmental protection system for the people's Republic of China. Organ Stud 2007;28:1013-34.

34. Greenwood R, Suddaby R, Hinings CR. Theorizing change: the role of professional associations in the transformation of institutionalized fields. Acad Manag J 2002;45:58-80.

35. Fligstein N. Social skill and institutional theory. Am Behav Sci 1997; 40:397-405

36. Lawrence TB. Institutional strategy. J Manag 1999;25:161-87.

37. Rao H, Morrill C, Zald MN. Power plays: how social movements and collective action create new organizational forms. Res Organ Behav 2000;22:237-81.

38. Perkmann M, Spicer A. 'Healing the scars of history': projects, skills and field strategies in institutional entrepreneurship. Organ Stud 2007;28:1101-22.

39. Keeney S, Hasson F, Mckenna HP. The Delphi technique in nursing and health research. Ames, lowa, Chichester, West Sussex, UK: Wiley-Blackwell, 2011.

40. Brennan N, Bryce M, Pearson M, et al. Understanding how appraisa of doctors produces its effects: a realist review protocol. BMJ OPEN 2014:4:e005466.

41. Pearson M, Brand SL, Quinn C, et al. Using realist review to inform intervention development: methodological illustration and conceptual platform for collaborative care in offender mental health. Implement Sci 2015;10:134.

42. Bazeley P, Jackson K. Qualitative data analysis with NVivo. Vol. Secondition. Los Angeles: SAGE, 2013.

43. Pace R, Pluye $P$, Bartlett $G$, et al. Testing the reliability and efficiency of the pilot Mixed Methods Appraisal Tool (MMAT) for systematic mixed studies review. Int J Nurs Stud 2012;49:47-53.

44. Mertens DM. Transformative research and evaluation. New York: Guilford Press, 2009 